\title{
Nondiagonal Response of Si by Inelastic-X-Ray-Scattering Experiments at Bragg Position: Evidence for Bulk Plasmon Bands
}

\author{
W. Schülke and A. Kaprolat \\ Institute of Physics, University of Dortmund, D-4600 Dortmund, Germany
}

(Received 25 March 1991)

\begin{abstract}
Inelastic scattering of $\mathrm{x}$-ray photons from standing-wave fields is used to measure nondiagonal elements of the dielectric-response matrix of $\mathrm{Si}$. A peak-valley structure for $q<q_{c}$ is found, due to the fact that bulk plasmon bands, split near the zone boundary, contribute with different signs; hence, direct evidence for the existence of plasmon bands is obtained. Such detailed studies of the response matrix, together with the proposed plasmon-band model, offer a new access to dynamical screening in an inhomogeneous electron system.
\end{abstract}

PACS numbers: $71.45 . \mathrm{Gm}, 71.25 . \mathrm{Rk}, 78.70 . \mathrm{Ck}$

The interpretation of experimental studies of bulk plasmons is generally based on mean-field theory for the dielectric response [1]. Nevertheless, it is well known that local-field effects due to the lattice-periodic electron density of solids may influence the plasmon properties $[2,3]$. One important consequence of lattice periodicity should be the occurrence of plasmon bands and the formation of plasmon band gaps [4]. Although there exist quantitative estimations of band effects for bulk plasmons $[3,5]$, no experimental evidence for these effects in real crystalline solids has been given so far.

We offer in this Letter direct experimental evidence for the occurrence of bulk plasmon bands, by making use of inelastic scattering of $\mathrm{x}$-ray photons from standing-wave fields [coherent inelastic $x$-ray scattering (CIXS)]. It has been predicted by one of us [6] that CIXS should provide the unique possibility to obtain direct experimental information about nondiagonal elements of the dielectricresponse matrix. We will discuss our results in terms of a

plasmon-band model, which is described in the beginning of this Letter.

In a homogeneous electron system the energy $\hbar \omega_{0}$ of a bulk plasmon satisfies a dispersion relation of the form

$$
\hbar \omega_{0}(q)=\hbar \omega_{p}+\beta q^{2},
$$

where both the free-electron plasmon frequency $\omega_{p}$ and the constant $\beta$ depend on the electron density. In an inhomogeneous electron system with a lattice-periodic density, the plasmon frequency $\omega(\mathbf{q})$ is periodic in reciprocal space. Hence, the dispersion relation $\omega(\mathbf{q})$ can be represented in a bulk plasmon band structure $\omega_{v}\left(\mathbf{q}_{r}\right)[3,4]$ by backfolding $\omega(\mathbf{q})$ into the first Brillouin zone using a reciprocal-lattice vector $\mathbf{g}_{r}$, so that $\mathbf{q}_{r}=\mathbf{q}-\mathbf{g}_{r} . \quad v$ stands for the band index. This formal representation obtains physical significance when the interaction of plasmons is taken into account. According to Nozieres and Pines [7], the interaction of a $\mathbf{q}$ plasmon with a $\mathbf{q}+\mathbf{g}$ plasmon is mediated by the gth Fourier transform of the electron density, $\rho(\mathbf{g})$, and can be represented by the Hamiltonian

$$
H_{i}=\sum_{\mathbf{g}}\{M(|\mathbf{q}|) M(|\mathbf{q}+\mathbf{g}|) / 2 m\} \rho(\mathbf{g}) \mathbf{q} \cdot(\mathbf{q}+\mathbf{g}) Q(\mathbf{q}) Q(-\mathbf{q}-\mathbf{g}),
$$

where $M(q)=4 \pi e^{2} / q^{2}$. $Q(\mathbf{q})$ are collective plasmon coordinates, and all $\mathbf{q}+\mathbf{g}$ should be restricted to values smaller than the plasmon cutoff vector $q_{c}$. Let us now construct an "empty lattice" plasmon band structure by backfolding $\omega_{0}(q)$ of Eq. (1). Then by switching on the plasmon interaction according to Eq. (2), one obtains a system of linear eigenvalue equations,

$$
\left\{\omega_{0}\left(\mathbf{q}_{r}+\mathbf{g}\right)-\omega\left(\mathbf{q}_{r}\right)\right\} a\left(\mathbf{q}_{r}+\mathbf{g}\right)+\sum_{\mathbf{g}^{\prime}} B\left(\mathbf{q}_{r}, \mathbf{g}, \mathbf{g}^{\prime}\right) a\left(\mathbf{q}_{r}+\mathbf{g}^{\prime}\right)=0,
$$

whose eigenvalues $\omega_{v}\left(\mathbf{q}_{r}\right)$ form the plasmon band structure. The eigenvector components $a_{v}\left(\mathbf{q}_{r}+\mathbf{g}\right)$ can be interpreted as probability amplitudes, whose squared modulus determines the probability to find a plasmon with wave vector $\mathbf{q}_{r}+\mathbf{g}$ at energy $\hbar \omega_{v}\left(\mathbf{q}_{r}\right)$. The coefficients

$$
B\left(\mathbf{q}_{r}, \mathbf{g}, \mathbf{g}^{\prime}\right)=\left\{M\left(\left|\mathbf{q}_{r}+\mathbf{g}\right|\right) M\left(\left|\mathbf{q}_{r}+\mathbf{g}^{\prime}\right|\right) / 4 m\right\} \operatorname{Re} \rho\left(\mathbf{g}^{\prime}-\mathbf{g}\right)\left\{\left(\mathbf{q}_{r}+\mathbf{g}\right) \cdot\left(\mathbf{q}_{r}+\mathbf{g}^{\prime}\right) / \omega_{0}\left(\mathbf{q}_{r}+\mathbf{g}^{\prime}\right)\right\}
$$

define the magnitude of the energy gaps. Equations (3) and (4) constitute what we call the plasmon-band model.

Now let us assume that $\mathrm{x}$ rays, scattered by a lattice-periodic electron system, excite only collective modes with $\delta$ function-like energy distributions. According to Fermi's "golden rule," we obtain the double-differential scattering cross section (DDSCS)

$$
d^{2} \sigma / d \omega d \Omega=r_{0}^{2}\left(\omega^{\prime} / \omega_{0}\right)\left(\hat{\mathbf{e}}_{0} \cdot \hat{\mathbf{e}}^{\prime}\right)^{2} \sum_{v}\left|a_{v}\left(\mathbf{q}_{r}+\mathbf{g}_{r}\right)\right|^{2} \delta\left(\omega-\omega_{v}\left(\mathbf{q}_{r}\right)\right)
$$

if momentum $\mathbf{q}_{r}+\mathbf{g}_{r}$ is transferred and an energy shift $\hbar \omega$ is observed. $\hat{\mathbf{e}}_{0}, \omega_{0}$ and $\hat{\mathbf{e}}^{\prime}, \omega^{\prime}$ are the polarization vectors and frequencies of the incident and the scattered photons, respectively. $r_{0}$ is the classical electron radius. Likewise we can 
express a general element of the dielectric-response matrix in terms of the plasmon-band model, when, following Ref. [4],

$$
\operatorname{Im} \varepsilon^{-1}\left(\mathbf{q}_{r}+\mathbf{g}, \mathbf{q}_{r}+\mathbf{g}^{\prime}, \omega\right)=-4 \pi^{2} e^{2}\left(\left|\mathbf{q}_{r}+\mathbf{g}\right|\left|\mathbf{q}_{r}+\mathbf{g}^{\prime}\right|\right)^{-1} \sum_{v} a_{v}\left(\mathbf{q}_{r}+\mathbf{g}\right) a_{v}^{*}\left(\mathbf{q}_{r}+\mathbf{g}^{\prime}\right) \delta\left(\omega-\omega_{v}\left(\mathbf{q}_{r}\right)\right) .
$$

This relation will be fundamental for the interpretation of our experiments.

Equation (5) suggested how to find experimental evidence for the plasmon band structure: We had to measure the DDSCS for a momentum transfer of $\mathbf{q}=\mathbf{g}_{s} / 2$, where $\mathbf{g}_{s}$ is the shortest reciprocal-lattice vector for which $\rho(\mathbf{g}) \neq 0$, so that, within the limits of a two-plasmon-band model, the squared eigenvector components of the two bands, $\left|a_{1,2}\left(\mathbf{g}_{s} / 2\right)\right|^{2}$, are of equal size. Under these experimental conditions we should expect a double-peak structure in the DDSCS with peaks at $\omega_{1,2}\left(\mathbf{g}_{s} / 2\right)$ according to Eqs. (4)-(6), separated by the gap energy $2 \hbar B\left(\mathbf{g}_{s} / 2,0, \mathbf{g}_{s}\right)$. Additionally we had to take care that $g_{s} / 2$ was smaller than the plasmon cutoff vector $q_{c}$. These conditions could be satisfied for $\mathrm{Si}\left(q_{c}=0.65\right.$ a.u. $)$ by $\mathbf{g}_{s}=(2 \pi / a)(1,1,1)$, as first pointed out by Oliveira and Sturm [5]. But we failed to detect the double-peak structure when performing a conventional inelasticscattering experiment with $q=(\pi / a)(1,1,1)$, which was not surprising, since a band gap of $1.1 \mathrm{eV}$ [5] is facing a plasmon linewidth of $5 \mathrm{eV}$.

An alternative way to obtain experimental evidence for plasmon bands is suggested by Eq. (6) and consists of looking for experimental information about $\operatorname{Im} \varepsilon^{-1}\left(\mathrm{~g}_{s} / 2\right.$, $\left.-\mathbf{g}_{s} / 2, \omega\right)$, a special nondiagonal element of the dielectric-response matrix. Within the limits of a twoplasmon-band model, the corresponding products $a_{1,2}\left(\mathbf{g}_{s} /\right.$ 2) $a_{1,2}^{*}\left(-\mathbf{g}_{s} / 2\right)$ of eigenvector components are equal in size in both bands, but positive in the lower-lying ( $p$ type) band and negative in the upper ( $s$-type) band, assuming $\rho(\mathbf{g})>0$. Therefore, if by any experiment the nondiagonal response could be measured, the corresponding spectra should exhibit a peak-valley fine structure, which should be detected more easily than a double-peak structure. This is the line we have followed in our experiment.

Information about nondiagonal response is equivalent to insight into local-field effects of the dielectric response. This kind of information can be obtained only by means of an experiment which is spatially selective rather than spatially averaging. Conventional inelastic-scattering experiments are spatially averaging due to the fact that the primary photon state is a (nearly) plane wave, which excites every spatial position with equal probability. It has been pointed out by one of us [6] that spatial selectivity, and therefore access to nondiagonal response, can be achieved by using a standing-wave field to act as a primary photon state of an inelastic-x-ray-scattering experiment. The experimental realization is shown in Fig. 1. Synchrotron radiation from the DORIS storage ring is monochromatized to an energy of $7990 \mathrm{eV}$ by means of a plane double-crystal monochromator. A standing-wave

field with the required intensity distribution

$$
I(\mathbf{r}, y)=A^{2}(y)+B^{2}(y)+2 A(y) B(y) \cos [\mathbf{g} \cdot \mathbf{r}+\Delta \phi(y)]
$$

has been obtained within the nearly perfect $\mathrm{Si}$ scattering sample as the result of coherent superposition of an incident plane wave with a Bragg-reflected wave, where the Bragg reflection may be characterized by the reciprocallattice vector $g$. The partial-wave amplitudes $A$ and $B$, as well as their mutual phase shift $\Delta \phi$, are strongly varying functions of a generalized incidence parameter $y$ (see Ref. [8]), which is +1 and -1 , respectively, at the bounds of the Bragg total reflection range. With the experimental setup, as sketched in Fig. 1, this incidence parameter can be varied and is completely determined by the angular position of the sample crystal relative to the second monochromator crystal, since both crystals constitute a nondispersive double-crystal setting. Therefore, the incidence parameter $y$ can be controlled by the "position" on the so-called rocking curve, which is the Braggreflected intensity (recorded by detector 1) as a function of the relative angular position of both crystals, as shown in greater detail elsewhere [8]. Especially, the phase shift $\Delta \phi$ changes from 0 to $\pi$, when going from one flank of the rocking curve to the opposite one. The scattered photons are energy analyzed in the so-called inverse geometry [9] by means of the spherically bent $\mathrm{Si}(777)$ crystal analyzer and recorded by detector 2 . Thus we are measuring the DDSCS for scattering off a standing-wave field with 2.5$\mathrm{eV}$ energy resolution and a $\mathrm{q}$ resolution of $\Delta q / q= \pm 0.1$.

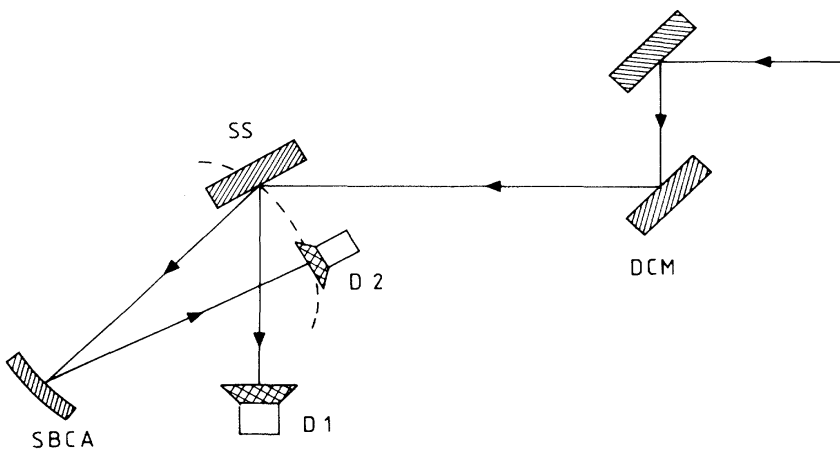

FIG. 1. Experimental setup: DCM, Si(111) double-crystal monochromator; SS, Si sample crystal; SBCA, spherically bent crystal analyzer; D1, detector 1 (for Bragg-reflected photons); D2, detector 2 (for energy-analyzed radiation). Note that in general the Bragg-diffraction plane of SS does not coincide with the Bragg-diffraction plane of SBCA. 


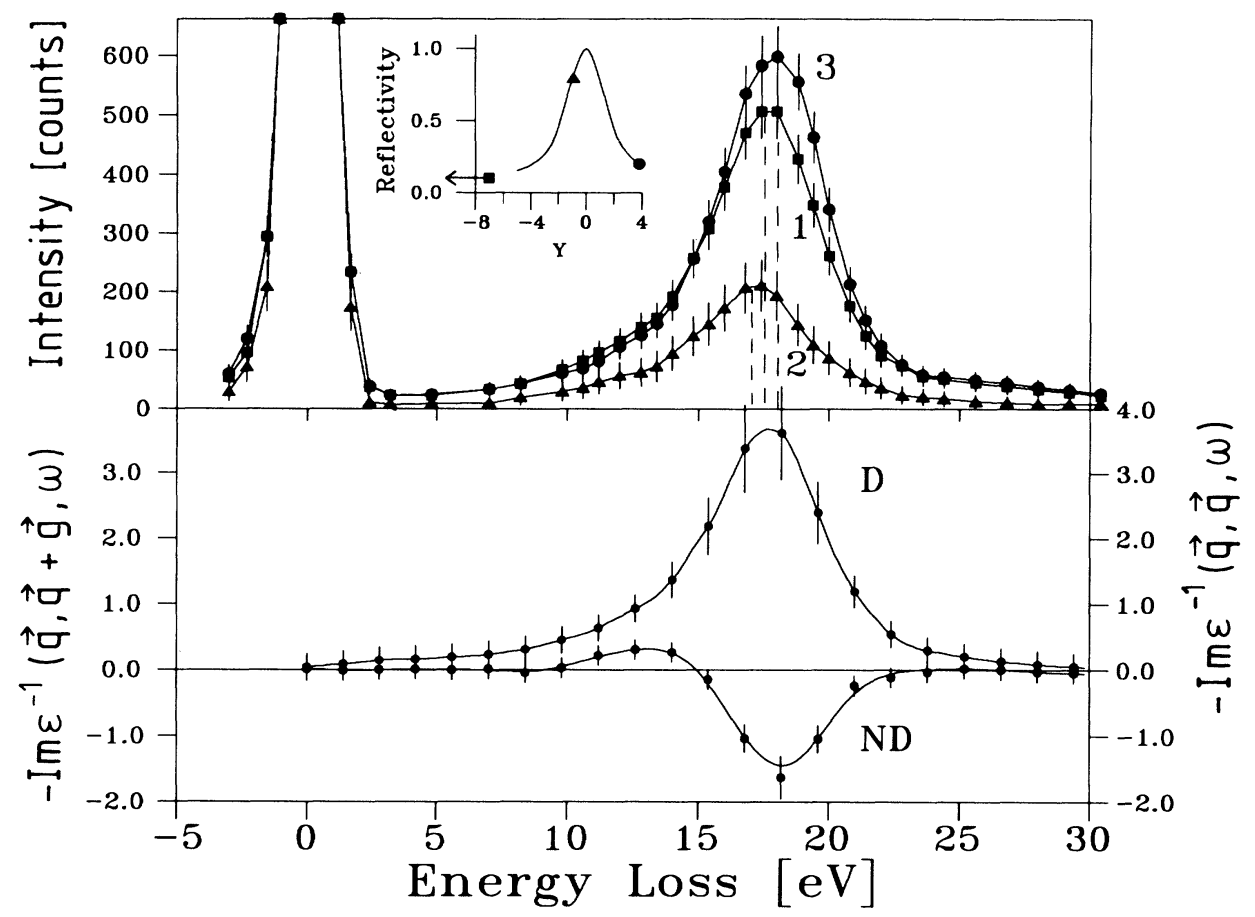

FIG. 2. Upper part: Rough experimental spectra (including the bottom of the quasielastically scattered line) of Si with $|\mathbf{q}|=0.51$ a.u. for three different positions on the rocking curve as indicated in the inset. Vertical broken lines indicate the centers of the corresponding spectra. Lower part: One diagonal term (D) and the nondiagonal term (ND) of the response matrix obtained from the above data by means of a separation procedure [8] and an interpolation to constant data-point distance.

The exact expression for the corresponding DDSCS has been derived in Ref. [8] and is specified here for the case of a centrosymmetric crystal structure:

$$
\begin{aligned}
d^{2} \sigma / d \omega d \Omega=-r_{0}^{2}\left(\omega^{\prime} / 4 \pi^{2} e^{2} \omega_{0}\right)[ & A^{2}(y)\left(\hat{\mathbf{e}}_{0} \cdot \hat{\mathbf{e}}^{\prime}\right)^{2}\left|\mathbf{q}_{r}+\mathbf{g}_{r}\right|^{2} \operatorname{Im} \varepsilon^{-1}\left(\mathbf{q}_{r}+\mathbf{g}_{r}, \mathbf{q}_{r}+\mathbf{g}_{r}, \omega\right) \\
& +B^{2}(y)\left(\hat{\mathbf{e}}_{h} \cdot \hat{\mathbf{e}}^{\prime}\right)^{2}\left|\mathbf{q}_{r}+\mathbf{g}^{\prime}\right|^{2} \operatorname{Im} \varepsilon^{-1}\left(\mathbf{q}_{r}+\mathbf{g}^{\prime}, \mathbf{q}_{r}+\mathbf{g}^{\prime}, \omega\right) \\
& \left.+2 A(y) B(y) \cos \Delta \phi(y)\left(\hat{\mathbf{e}}_{0} \cdot \hat{\mathbf{e}}^{\prime}\right)\left(\hat{\mathbf{e}}_{h} \cdot \hat{\mathbf{e}}^{\prime}\right)\left|\mathbf{q}_{r}+\mathbf{g}_{r}\right|\left|\mathbf{q}_{r}+\mathbf{g}^{\prime}\right| \operatorname{Im} \varepsilon^{-1}\left(\mathbf{q}_{r}+\mathbf{g}_{r}, \mathbf{q}_{r}+\mathbf{g}^{\prime}, \omega\right)\right],
\end{aligned}
$$

where $\hat{\mathbf{e}}_{h}$ is the polarization vector of the Bragg-reflected plane wave, and $\mathbf{g}^{\prime}$ is defined by $\mathbf{g}^{\prime} \equiv \mathbf{g}_{r}-\mathbf{g}$. By means of Eq. (6) we can introduce the plasmon-band model into Eq. (8) and find that the interference term of the DDSCS contains the desired information about terms of the form $a_{v}\left(\mathbf{q}_{r}+\mathbf{g}\right) a_{v}^{*}\left(\mathbf{q}_{r}+\mathbf{g}^{\prime}\right)$, which will give rise to a peakvalley fine structure at the zone boundary as pointed out above, and can offer evidence for plasmon banding.

In the upper part of Fig. 2 exemplarily measured spectra for three different positions on the rocking curve (as indicated in the inset) are shown. The difference in height of these spectra is mainly due to the strongly $y$ dependent absorption and extinction. The influence of the interference term of Eq. (8) can be demonstrated by comparing curve 1 , corresponding to an angular position of the sample far away from the center of the rocking curve $(y=-1500)$, and hence only determined by diagonal terms of the response matrix (shown as curve $D$, after separation, in the lower part of Fig. 2), with curve 2 (3), corresponding to $\Delta \phi(y) \approx 0(\approx \pi)$ and for that reason containing the relevant nondiagonal term (shown as curve ND in the lower part of Fig. 2) with positive (negative) sign. Hence, the peak-valley structure of the nondiagonal term ND leads the center of curve 2 (3) being shifted to lower (higher) energy loss when compared with curve 1. After subtraction of both the quasielastically scattered line (see the upper part of Fig. 2) and the background we have separated out the interference term according to Ref. [8] by measuring relative values of the DDSCS at altogether seven positions on the rocking curve. The final results are put on an absolute scale by using the $f$-sum rule [10]. In Figs. 3(a) and 3(b) experimental nondiagonal elements of the $\mathrm{Si}$ response matrix for $\mathrm{g}=(2 \pi / a)$ $\times(\overline{1}, \overline{1}, \overline{1})$ and two different scattering vectors $\mathbf{q} \equiv \mathbf{q}_{r}$ are presented, where $q \leq q_{c}$. As shown in Fig. 3 both q's point to the (111) Brillouin-zone boundary. Therefore, the lowest two plasmon bands around the $L$ point should 

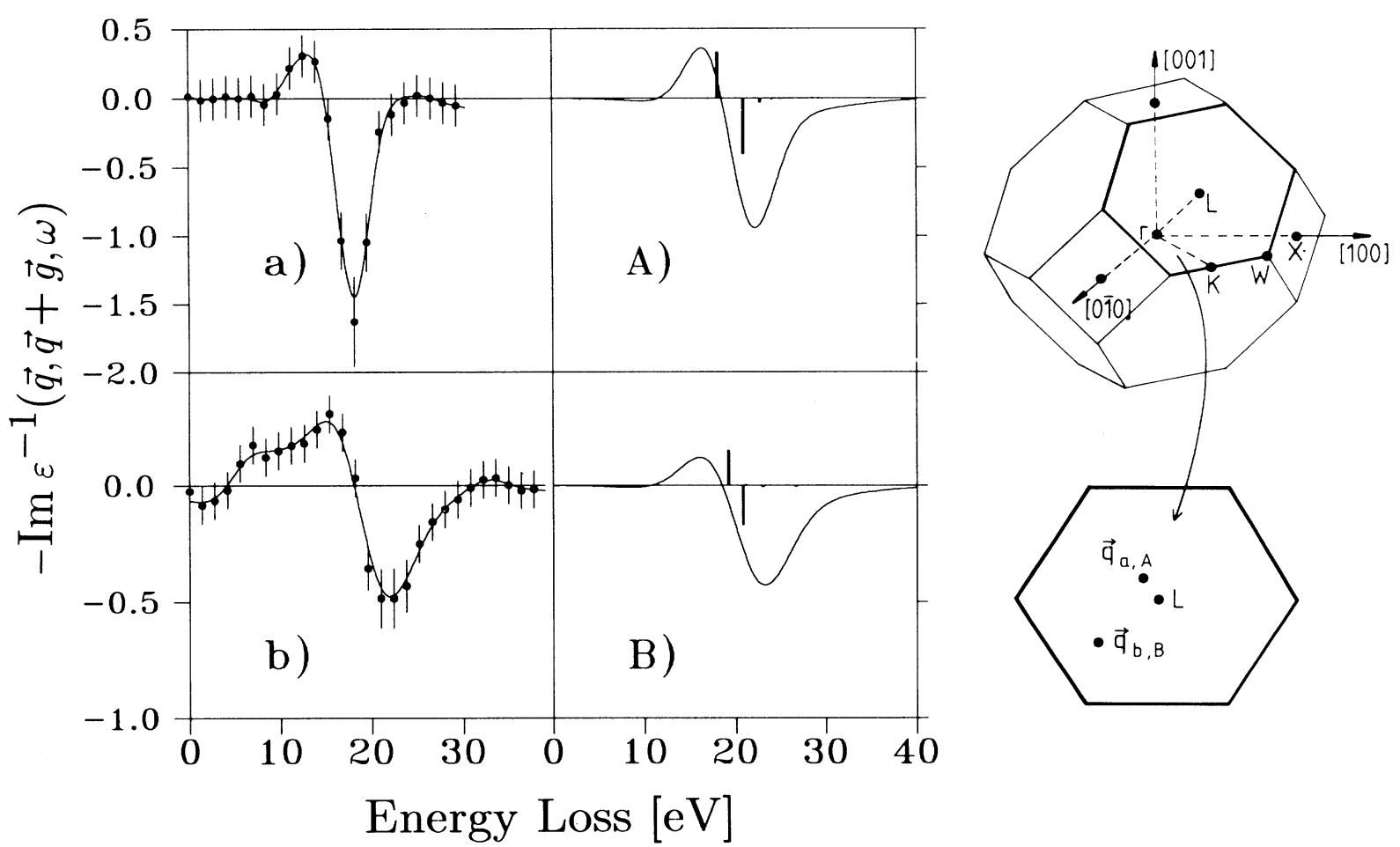

FIG. 3. Left-hand part: (a),(b) Experimental and (A),(B) corresponding model-calculated nondiagonal elements of the response matrix, $\operatorname{Im} \varepsilon^{-1}(\mathbf{q}, \mathbf{q}+\mathbf{g}, \omega)$, with $\mathbf{g}=(2 \pi / a)(\overline{1}, \overline{1}, \overline{1}),\left|\mathbf{q}_{(\mathrm{a}) \text {, (A) }}\right|=0.51$ a.u., $\left|\mathbf{q}_{(\mathrm{b}),(\mathrm{B})}\right|=0.64$ a.u.; the end points of both $\mathbf{q}_{(\mathrm{a}) \text {,(A) }}$ and $\mathbf{q}_{(\mathrm{b}),(\mathrm{B})}$ are on the (111) Brillouin-zone boundary, as shown in the right-hand part. The vertical bars in (A) and (B) indicate the energy position of the $\delta$-function-like plasmon resonances of the plasmon-band model. Right-hand part: Position of the $\mathbf{q}$ end points on the Brillouin-zone boundary.

be excited with nearly equal strength, so that a peakvalley structure of the relevant nondiagonal element is expected within the limits of the plasmon-band model, as pointed out above. This is exactly what we found experimentally. The qualitative aspects of the fine structure are confirmed by calculations according to the plasmon-band model, shown in Figs. 3(A) and 3(B). The plasmon dispersion $\omega_{0}(q)$ used is fitted to electron data [11] $\left(\omega_{p, e}=17.96 \mathrm{eV}, \quad \beta_{e}=6.45 \mathrm{eV} /\right.$ a.u. $) ; \quad \rho(111) / \rho(000)$ $=0.223$ is from experiment [12]. The vertical bars in Figs. 3(A) and 3(B) apply to a $\delta$-function-like energy distribution for each plasmon resonance [see Eq. (6)], and the solid curves correspond to Gaussian distributions, whose width $\Delta \omega(q)$ has been fitted to empirical x-ray data [13] $\left[\Delta \omega(q)=0.6+18.5 q^{2} \mathrm{eV}, q\right.$ in a.u.]. The first frequency moments of the experimental nondiagonal terms fulfill the Johnson sum rule [10] within experimental error.

In summary we have shown that CIXS offers evidence for the existence of plasmon bands by measuring nondiagonal elements of the dielectric-response matrix, and presents detailed information about the response matrix, which enables us to handle better the problem of dynamical screening in an inhomogeneous electron system.

This work has been funded by the German Federal Ministry of Research and Technology (BMFT) under
Contract No. 05434 AXB.

[1] H. Ehrenreich and M. H. Cohen, Phys. Rev. 115, 786 (1959).

[2] S. G. Louie, J. R. Chelikowsky, and M. L. Cohen, Phys. Rev. Lett. 34, 155 (1975).

[3] K. C. Pandey, P. M. Platzman, P. Eisenberger, and E-Ni Foo, Phys. Rev. B 9, 5046 (1974).

[4] W. M. Saslow and G. F. Reiter, Phys. Rev. B 7, 2995 (1973).

[5] L. E. Oliveira and K. Sturm, Phys. Rev. B 22, 6283 (1980).

[6] W. Schülke, Solid State Commun. 43, 863 (1982); 44, 1130 (1982).

[7] P. Nozieres and D. Pines, Phys. Rev. 109, 741 (1958).

[8] W. Schülke and S. Mourikis, Acta Crystallogr. Sect. A 42, 86 (1986).

[9] W. Schülke and H. Nagasawa, Nucl. Instrum. Methods Phys. Res., Sect. A 222, 203 (1984).

[10] D. L. Johnson, Phys. Rev. B 9, 4475 (1974).

[11] J. Stiebling and H. Raether, Phys. Rev. Lett. 40, 1293 (1978).

[12] T. Saka and N. Kato, Acta Crystallogr. Sect. A 42, 469 (1986).

[13] J. R. Schmitz, A. Kaprolat, and W. Schülke (to be published). 